\title{
SPE-195020-MS
}

\section{Validation of High Pressure Resin Impregnation Technique for High Resolution Confocal Imaging of Geological Samples}

\author{
A. Hassan, King Abdullah University of Science \&Technology (KAUST); M. P. Yutkin, KAUST; V. Chandra, KAUST; T. W. Patzek, KAUST
}

Copyright 2019, Society of Petroleum Engineers

This paper was prepared for presentation at the Middle East oil \& gas exhibition and conference held in Bahrain, 18-21 March 2019.

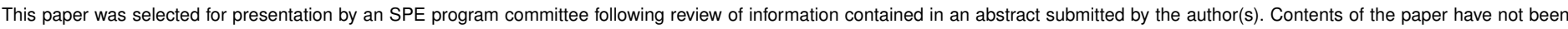

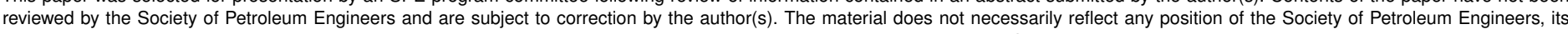

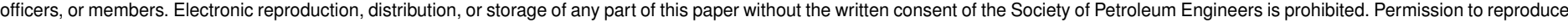
in print is restricted to an abstract of not more than 300 words; illustrations may not be copied. The abstract must contain conspicuous acknowledgment of SPE copyright.

\section{Abstract}

In this paper, we present a procedure for high pressure resin impregnation of microporous rock. This procedure produces the highquality pore casts that reveal the fine details of the complex pore space of micritic carbonates. We carefully test our resin impregnation procedure and demonstrate that it renders the high resolution, 3D confocal images of pore casts. In our work, we use silicon micromodels as a reference to validate the key parameters of high-pressure resin impregnation. We demonstrate possible artifacts and defects that might develop during rock impregnation with resin, e.g., the resin shrinkage and gas trapping. The main outcome of this paper is a robust protocol for obtaining the high-quality epoxy pore casts suitable for rock imaging with Confocal Laser Scanning Microscopy (CLSM). We have implemented this protocol and provided the high resolution, three-dimensional (3D) imagery and description of microporosity in micritic carbonates.

\section{Introduction}

Saturation of permeable rock samples with a pore-filling material, e.g., epoxy, that provides a contrast between the optical and/or electric properties of the void space and mineral grains has been reported by several researchers, for example (Waldo and Yuster, 1937, Pittman and Duschatko, 1970, Wardlaw, 1976, Yanguas and Paxton, 1986, Klaver et al., 2015, Jobe et al., 2018). The pore-filling epoxy resin supports fragile pores at different stages of thin-section preparation, such as grinding or polishing. Also, optical contrast between the dyed epoxy resin and rock helps in pore identification. For geological samples, epoxy resin casting is typically used to preserve the mechanical integrity of the sample after polishing it down to 15 microns. Blue dye enables porosity counting, and fluorescent dyes have been getting more attention recently in combination with confocal imaging. Several studies have reported applications of epoxy pore casts to gain better understanding of fluid flow capacity of carbonate rocks (Cantrell et al., 1999, Morrow and Buckley, 2006, Fullmer et al., 2014). Despite the broad importance of rock saturation with epoxy resin, currently there exists no standard approach for high pressure pore casting. The impregnation procedure in general consists of three steps: i) resin preparation, ii) rock sample degassing, and, and iii) resin injection into the sample. Depending on the subsequent characterization method, these three steps may differ significantly. For example, for optical microscopy a dye must be mixed with the resin, while electron microscopy does not require a dye. For confocal laser microscopy, an additional step of etching the rock is required to obtain the 3D rock images (Hassan et al., 2017). In this work, we have critically evaluated and optimized the resin impregnation procedure that generates the high-quality epoxy pore casts for high resolution 3D imaging with CLSM.

\section{Resin impregnation}

Vacuum impregnation is the most widely adopted method for producing thin sections of geological samples (Minoura and Conley, 1971) and other porous materials, such as cement (John, 1990). Alternative methods, such as high pressure impregnation and epoxysolvent replacement are also adopted for rock analysis (Wardlaw, 1976, Smith and Anderson, 1995). The preference for the vacuum process stems from is its low cost and general availability of laboratory equipment.

Although vacuum impregnation is a simple and efficient method for preparation of the standard thin sections of rock, it has a number of limitations when the samples are too tight or microporous. Vacuum impregnation can only access a limited pore volume (Wong and Buenfeld, 2006), while excessive drying of the samples on vacuum may alter pore morphology (Hurst and Nadeau, 1995) or even induce cracks (Chen et al., 2002).

For vacuum impregnation of tight samples, Kjellsen et al. (2003) showed that the epoxy penetration depth is limited to $120 \mu \mathrm{m}$. Thus, studies focused on the characterization of tight samples employed high pressure resin impregnation in their methodology to ensure effective impregnation throughout the sample (Yanguas and Dravis, 1985, Gies et al., 1987, Fredrich, 1999, Shah et al., 2014). Beckett 
and Sellwood (1991) specified the parameters used to impregnate carbonate samples at high pressure generated by nitrogen gas. However, their description did not address any artifacts or defects that may emerge during the impregnation process. For example, the impact of dissolved gas in the epoxy mixture was not considered as a source of defects. Also, the vacuum level, at which the degassing step takes place prior to application of high pressure, has varied significantly in the literature, even for the same material. For a cementitious material, Chen et al. (2002) reported the 8 mbar vacuum level, while Kjellsen et al. (2003) reported 30 mbar. Camuti and McGuire (1999) and Boës and Fagel (2005) reported the vacuum level of 700 mbar and 800 mbar, respectively, for impregnation of unconsolidated samples. For rock samples, Beckett and Sellwood (1991) reported degassing under 1.33 mbar, while Soeder (1990) used $0.067 \mathrm{mbar}$. Kemp (1990) suggested using a very high vacuum level of $10^{-6}$ mbar before introducing a pre-evacuated epoxy.

An inherent property of epoxy resin is shrinkage after curing. This shrinkage has been considered as irrelevant or "negligible" in the earlier studies (Spurr, 1969, Minoura and Conley, 1971, Jim, 1985, Yadav et al., 1987, Soeder, 1990, Beckett and Sellwood, 1991). This may hold true for linear shrinkage determined according to the standard ASTM method, which is based on the resin volume variation before and after curing. We will show below that other types of shrinkage defects may develop, and unless prevented from occurring or properly identified in the samples, these defects may lead to false interpretations of the subsequent high resolution images.

Some studies, e.g. (Spurr, 1969, Minoura and Conley, 1971, Head and Buenfeld, 2006, Shah et al., 2014) suggested mixing solvents, such as acetone or toluene, with epoxy to decrease its viscosity for more efficient impregnation. We show that this practice may lead to improper results. Moreover, a reduction of the epoxy viscosity would only slightly improve impregnation efficiency.

All these variations and discrepancies in the resin impregnation procedure have resulted in the general lack of standard sample preparation protocol. In this study, we use the custom-fabricated micromodels with the well-defined pore geometry to validate the parameters of high-pressure resin impregnation. Based on these experiments, we try to address the above mentioned concerns and provide guidelines for obtaining the high-quality pore casts for high resolution imaging.

\section{Confocal imaging}

CLSM is an imaging technique that can increase the optical resolution and contrast of a micrograph by eliminating out-of-focus light. CLSM uses a laser light source that is tightly focused through a slit or pin-hole aperture, limiting the depth of field to a single plane. By varying the focal point, multiple images of different surface planes can be obtained to produce a sharply focused 3D image of the object (Rothwell and Rack, 2006). During CLSM imaging, true 3D resolution is thus accomplished by actively suppressing light signal coming from out-of-focus planes (Muller, 2006). CLSM can achieve resolutions of around 0.2 and $0.3 \mu \mathrm{m}$ in the XY and Z planes, respectively, by using high numerical aperture (NA) objectives and by minimizing the pinhole size (Pawley, 2010). However, the imaging depth of CLSM is small for nontransparent materials, because light scattering limits laser penetration and degrades the obtained fluorescence signal. CLSM penetrates as deeply as $7 \mu \mathrm{m}$ for concrete (Head and Buenfeld, 2006), 90-100 $\mu \mathrm{m}$ for sandstone (Fredrich et al., 1995, Petford et al., 2001), and $10 \mu \mathrm{m}$ for carbonates (Fredrich, 1999, Shah et al., 2014).

In this study, we use CLSM to image micromodels, which were impregnated under different conditions, and exemplify defects and artifacts that may emerge during gas-assisted high pressure resin impregnation. From the experiments on micromodels, we could optimize the parameters of the process for high-resolution 3D confocal imaging of pore space. We follow the rock characterization procedure presented in (Hassan et al., 2017), where the rock matrix in the fluorescent epoxy pore cast is dissolved using hydrochloric acid to allow relatively large depth of investigation of up to $300 \mu \mathrm{m}$. Implementing this approach, we were able to provide high-resolution 3D images of the microporosity present in carbonate samples.

\section{Results}

\section{Estimation of impregnation parameters}

The quality of the impregnated pore cast can be evaluated from the smallest feature size invaded by resin and the uniformity of resin throughout the sample. In order to assess the parameters that impact quality of the resin pore casts, the mechanisms governing resin impregnation should be addressed. Approximating micromodel channels with capillaries, the invading fluid progression is governed by capillarity, viscous drag, inertia and the imposed impregnation pressure, e.g., vacuum or positive impregnation pressure. Considering horizontal resin flow, where the hydrostatic pressure loss can be neglected, Washburn (1921) described the flow as

$$
\frac{d l}{d t}=\frac{R^{2}}{8 \mu l}\left(\frac{2 \gamma}{R} \cos \theta+P_{\text {impreg }}\right)
$$

where $\frac{d l}{d t}$ is the is the capillary penetration velocity, $R$ the tube radius, $\mu$ is the fluid viscosity, $\gamma$ the surface tension, $\theta$ is the advancing contact angle, and $P_{\text {impreg }}$ is the impregnation pressure imposed on the resin. The resin viscosity is time-dependent and can be approximated as

$$
\mu(t)=a e^{b t}
$$

where $t$ is time, and $a$ and $b$ are the constants experimentally measured to be $0.461 \mathrm{~Pa} \cdot \mathrm{s}$ and $10^{-4} \mathrm{~s}^{-1}$, respectively. 
Substituting Eq. (2) into Eq. (1), and integrating with the initial condition of $l=0$ at $t=0$ yields an estimation of resin penetration depth with time. The epoxy resin properties used in this model, its wettability and viscosity, were experimentally measured and summarized in Table 1. We also assume no change of wettability. Practically, the flow stops in the model when the resin polymer is too viscous to flow discernibly.

Fig. 1 shows the relative effect of the imposed impregnation pressure on the capillary penetration depth, according to the numerical solution of Eq. (1). The model assumes the single-phase resin flow in a capillary, which requires absolute vacuum to be achieved. The proposed model is a great simplification of the experimental system, yet it can provide useful insights into the impregnation process and its efficiency. A number of impregnation models has been proposed in the literature to address the limitations of this model (Szekely et al., 1971, Levine et al., 1976, Maija Leskinen, 1987, Marmur, 1992, Poulin et al., 1997, Hamraoui and Nylander, 2002).

The corollaries from this simple model are:

1. Resin should fill the pore space by spontaneous imbibition owing to its wetting behavior. This can be generalized to most of epoxy resins used in impregnation (Yamaki and Katayama, 1975, Wardlaw, 1976, Yadav et al., 1987, Li et al., 2006, Shah, 2014). The spontaneous imbibition usually never happens in practice because of limited impregnation time.

2. Application of high pressure helps in resin impregnation since significant pressures can be generated at laboratory scales, and

3. Together with pressure, viscosity reduction can improve the process. However, this approach has several drawbacks for highresolution imaging, as discussed below.

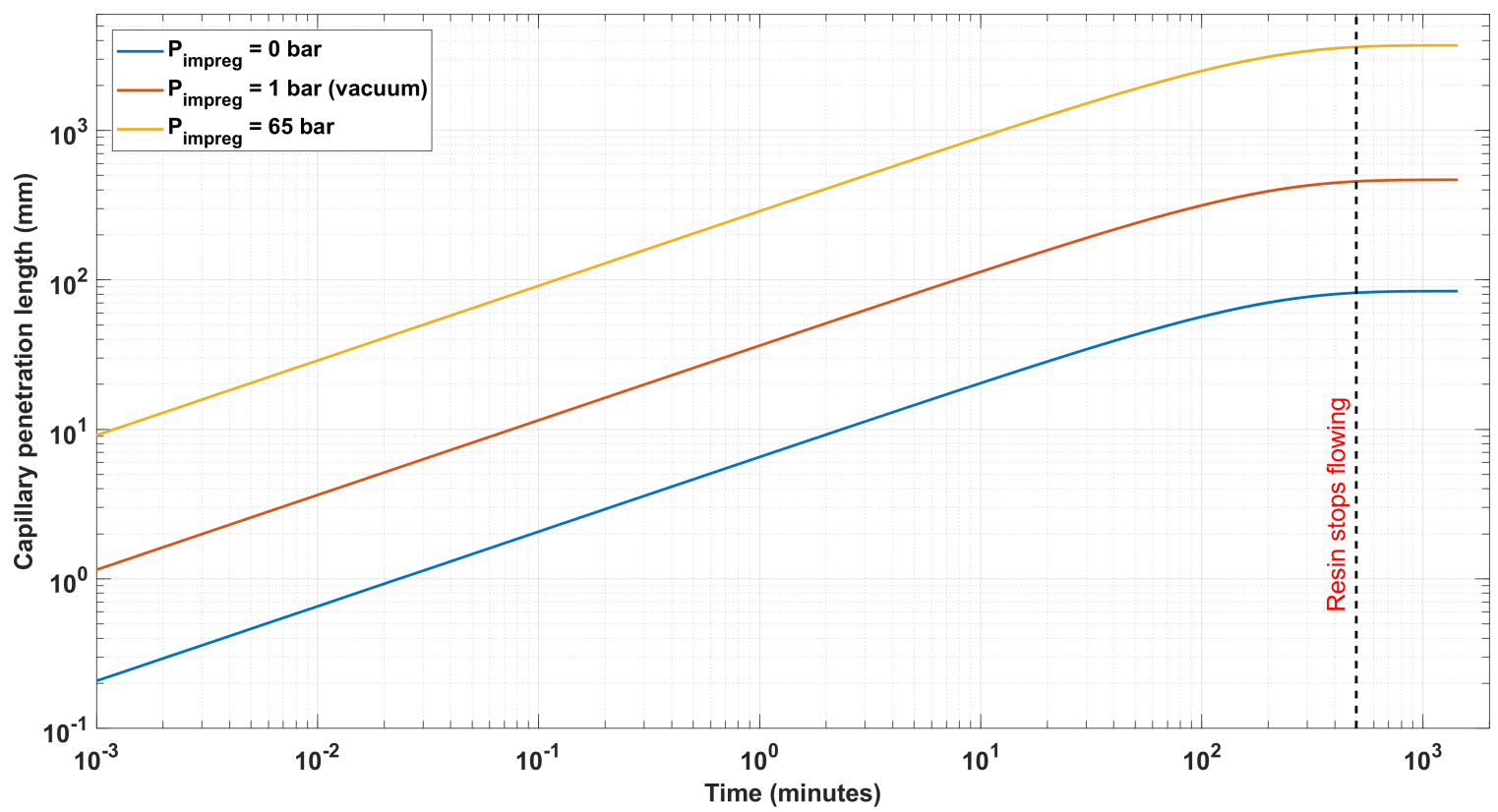

Figure 1: Visualization of Eq. (1) for $R=40 \mu \mathrm{m}$ at three different pressures: $P_{\text {impreg }}=0$ bar (case I), $P_{\text {impreg }}=1$ bar (case II, vacuum), and $P_{\text {impreg }}=65$ bar (case III). In case I, the flow is driven only by capillary forces, while in cases II and III the pressure on the resin is acting along with capillary forces to drive the flow. Assuming an infinite capillary, the penetration length in case III should be an order of magnitude higher than that in case II after the resin stops flowing.

\section{Factors controlling pore cast quality}

To reveal the factors that determine quality of resin impregnation, e.g., pressure, shrinkage and trapped gases, we have used the custom-fabricated models shown in Fig. A1. In contrast to the complex heterogeneous pore space of rocks, our micromodels have clearly defined simple pores, and even slight defects or artifacts from the resin impregnation procedure should be easily detected. The major classes of defects are classified in Table 2.

Vacuum level. In order to evaluate the efficiency of vacuum impregnation, we tested two scenarios: i) residual pressure of 120 mbar, and ii) residual pressure of 10 mbar, both shown in Fig. 2. Type 1 defects from the trapped gas (see the green annotation) are visible in the two models in Fig. 2. These defects have round or elliptical shapes and appear mostly near the channel ends. These defects are likely caused by the entrapment of gas in the micromodels by snap off, consistently with our observations at the lower vacuum level of 120 mbar, where type 1 defects were more abundant. 
Table 1: Epoxy resin properties

\begin{tabular}{lc}
\hline \multicolumn{2}{c}{ Epoxy resin } \\
\hline Density & $1118 \mathrm{~kg} / \mathrm{m}^{3}$ \\
Viscosity & $0.468 \mathrm{~Pa} \cdot \mathrm{s}$ \\
Surface tension & $0.039 \mathrm{~N} / \mathrm{m}$ \\
Contact-angle on silica & $31^{\circ}$ \\
Curing agent & Primary amine \\
Curing time & $24-36$ hours \\
\hline${ }^{*}$ The curing time is pressure dependent (see \\
\multicolumn{2}{c}{ discussion below) }
\end{tabular}

Table 2: Types of defects observed in resin impregnated micromodels

\begin{tabular}{ll}
\hline Defect type & Description \\
\hline Type 1 & Trapped gases \\
Type 2 & Shrinkage defects \\
Type 2A & Voids with corrugated boundaries, and irregular shapes \\
Type 2B & Rough cracks \\
\hline
\end{tabular}

Fig. 2 features type 2 defects (see the yellow annotation) that are clearly different from the type 1 defects. Usually type 2 defects are smaller, have corrugated boundaries and irregular shapes. Resin shrinkage is likely the cause of type 2 defects, which are frequently referred to as "volume decrease."

In resins, the two main causes of shrinkage are: i) thermal expansion and contraction due to heating or cooling, and ii) curing shrinkage due to polymer chain cross-linking (Ramos et al., 2005). In this study, we are only concerned with the curing shrinkage since epoxy cross-linking is taking place at room temperature.

Fig. 3 shows the two subtypes of shrinkage defects: i) type 2A: voids that are 10-40 $\mu \mathrm{m}$ in diameter, the most frequent type 2 defects, and ii) type 2B: large crack defects that are rare. Shrinkage defects are caused by tensile stresses that arise when resin is cured inside a constrained volume to which it adheres (Plepys and Farris, 1990, Eom et al., 2001) .

Shrinkage defects are more pronounced in Fig. 2a, where the high vacuum level, 10 mbar, was applied. Reaching 10 mbar in our setup requires significantly more time than reaching 120 mbar. Long vacuum exposure results in excessive evaporation of hardener despite added excess. The hardener deficiency causes a lower resin conversion ratio, slows down gelation, and generates resin inhomogeneity and voids.

Fig. 4 confirms that micromodels exposed to long degasing develop more type 2A defects. However, better degassing can be achieved by applying vacuum for a longer time. As a result, the trapped type 1 gas disappears. 

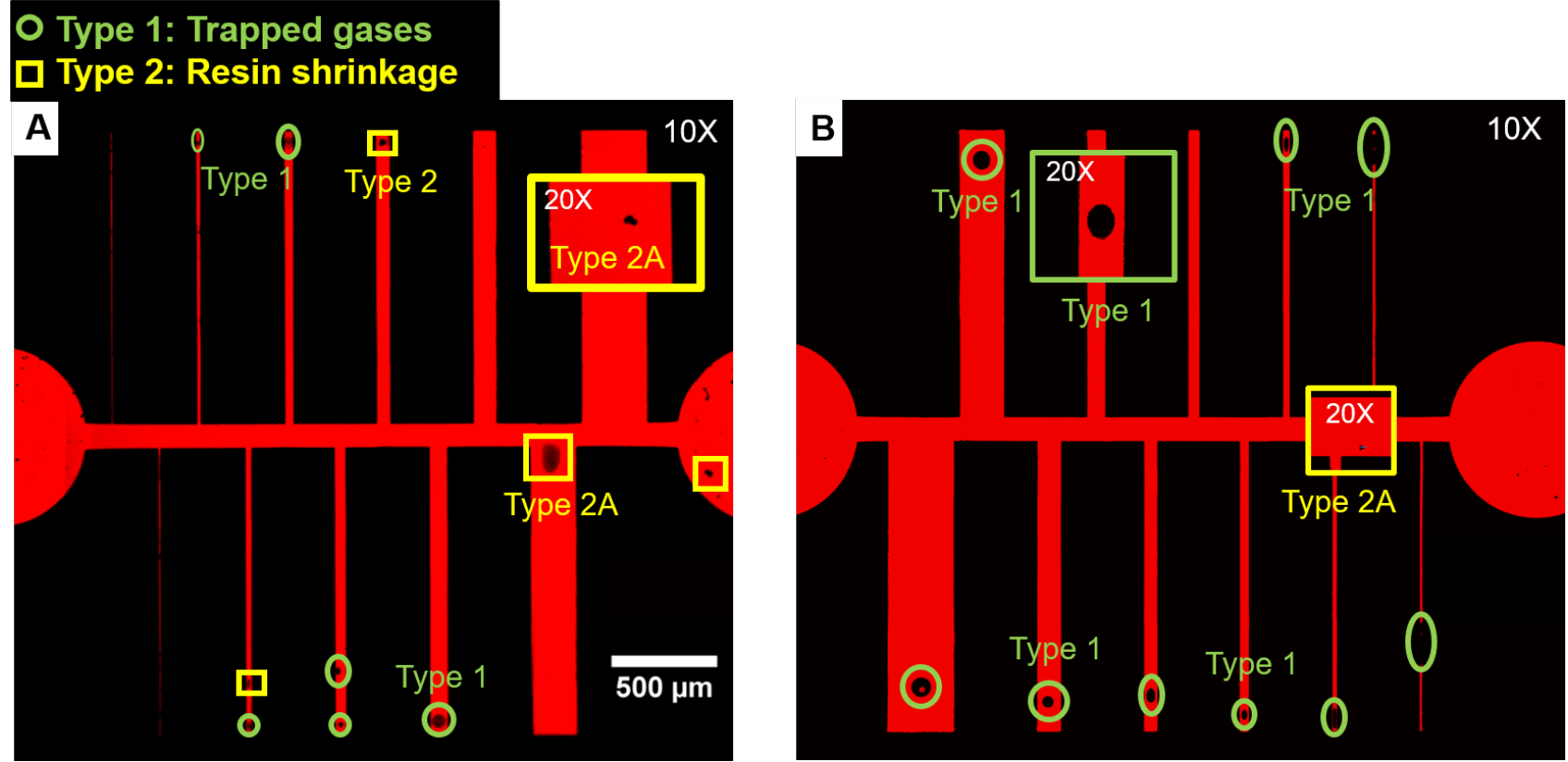

Figure 2: Confocal images of the micromodels impregnated by resin under two vacuum levels: (a) 10 mbar vacuum, and (b) 120 mbar vacuum. Type 1 defects, trapped gases, are more abundant in model B due to the inefficient degassing and impregnation energy. Type 2 shrinkage defects are more abundant in model $A$, because the epoxy resin is more aggressively vaporized. Type $2 A$ refers to void shrinkage defects while type $2 \mathrm{~B}$ refers to cracks.
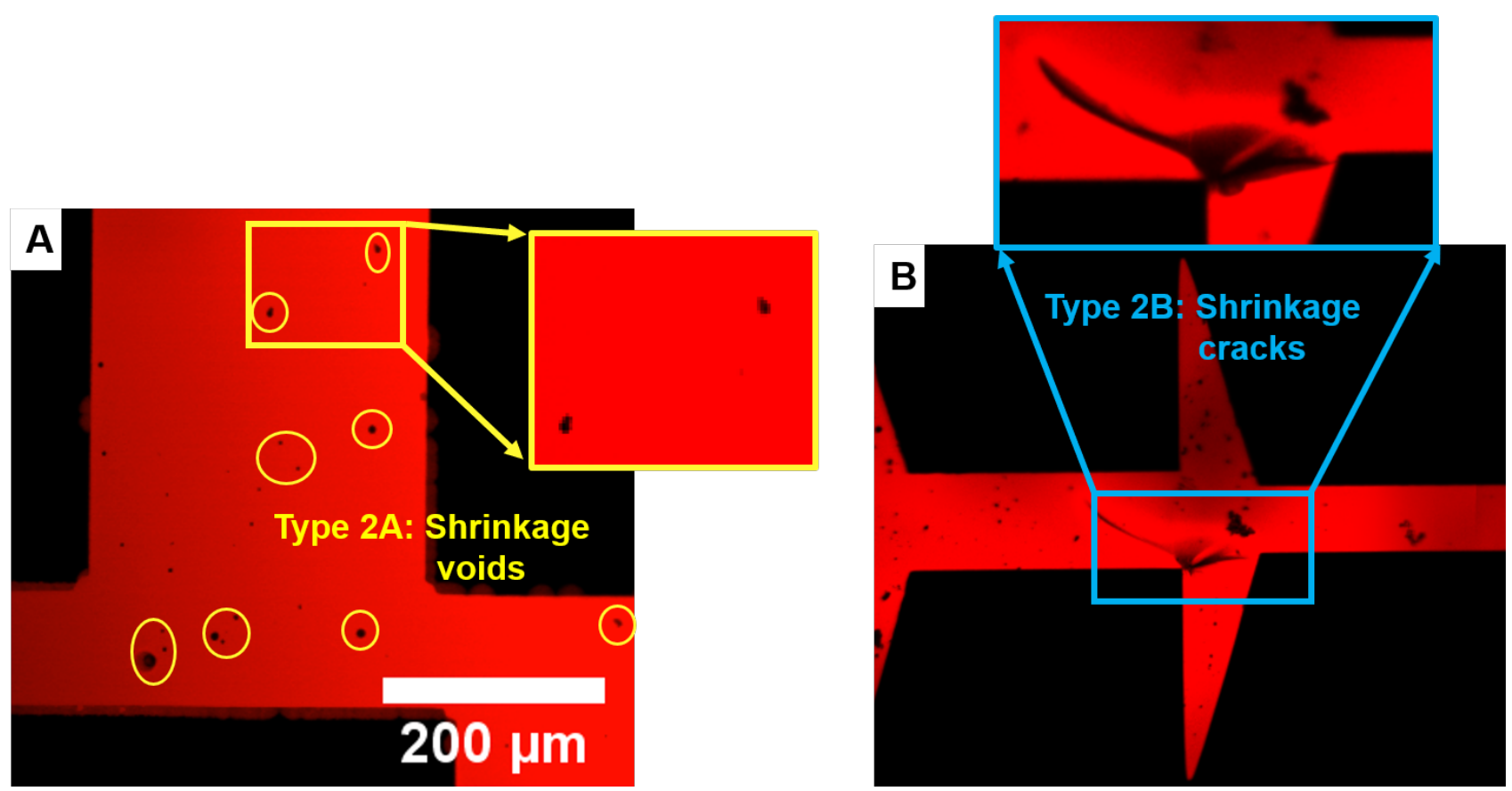

Figure 3: Confocal images of type 2 shrinkage defects: (A) the voids in the resin that have 10-40 $\mu \mathrm{m}$ size. (B) The rough shrinkage cracks that develop across the structure indicate high residual shrinkage stresses. 


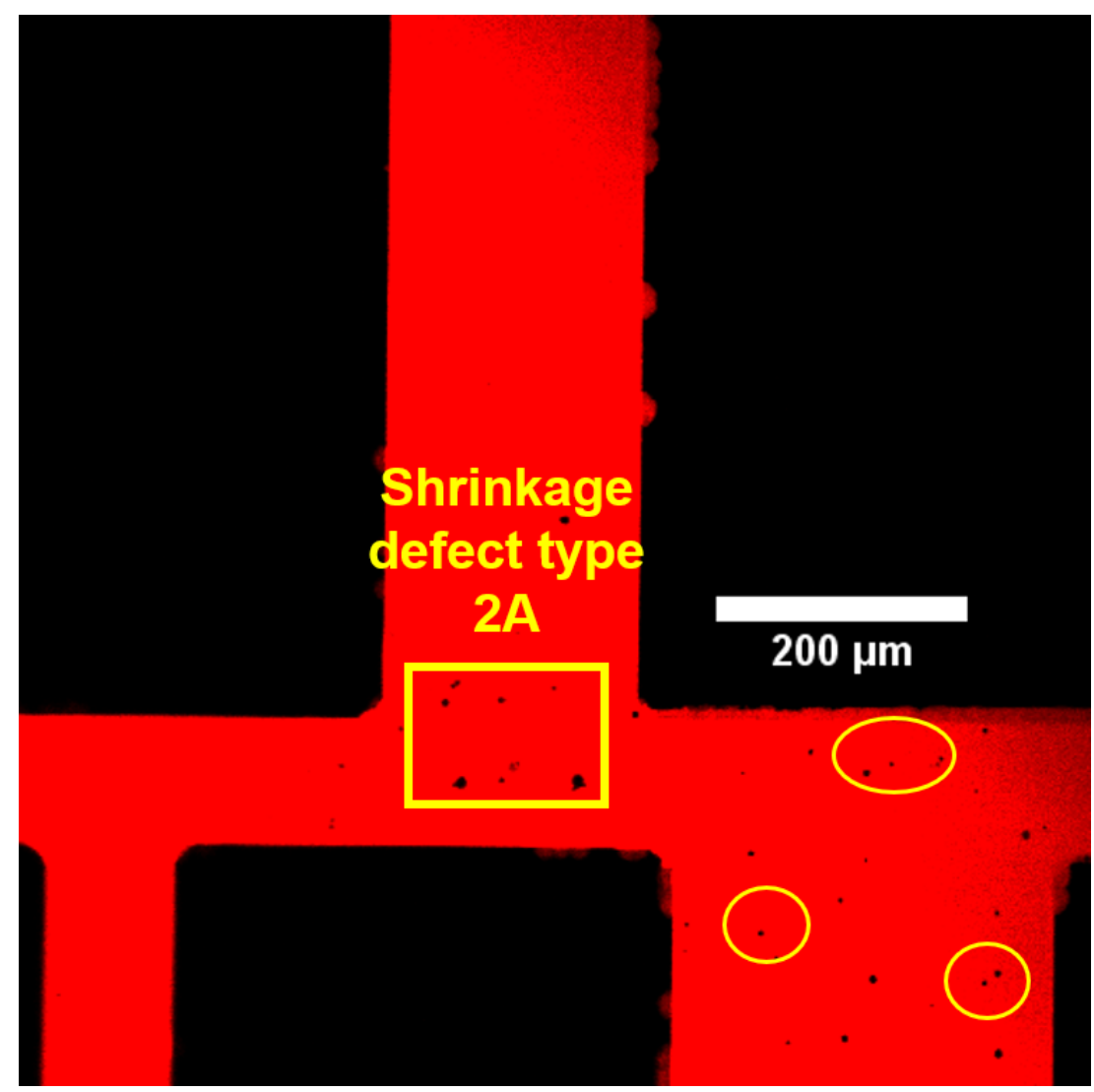

Figure 4: Confocal images of the micromodels vacuum-impregnated under 10 mbar for 20 minutes. Shrinkage defects, especially type $2 A$, are more abundant in this model, because the epoxy was vaporized for longer time.

Impregnation pressure. Fig. 5 shows the confocal images of two micromodels that were impregnated under: a) a high pressure preceded by degassing, and b) vacuum. We can clearly identify the trapped gas pockets inside the model that was impregnated under vacuum, see Fig. 5b. Even though the high pressure-assisted impregnation was proven to be more efficient, it is necessary to apply vacuum prior to high pressure in order to remove fluids from the sample. We tested this hypothesis by applying a pressure of 65 bar without the degassing step. Fig. 6 demonstrates the importance of the degassing step prior to the high pressure step. We observe gas bubbles without prior degassing even after applying 65 bar. The shrinkage defects, type $2 \mathrm{~A}$, are seen in both models in Fig. 6. These defects develop in most of the impregnated models and their severity depends on the vacuum level. In the case of high pressure, 65 bar, and degassing at vacuum level of 10 mbar or lower, shown in Fig. 3, the shrinkage defects are more abundant compared with the high pressure and degassing at 120 mbar shown in Fig. 6a.

High pressure does not only affect resin impregnation volume, but it controls the curing kinetics (Ramos et al., 2005) and the mechanical properties of the epoxy resin (Chen et al., 2002). Experimentally, this leads to increasing the curing time by almost 50\%. Releasing pressure before the complete curing, suggested by Beckett and Sellwood (1991), would lead to an abrupt release of the dissolved gas and the drastic results shown in Fig. 7. Another advantage of curing under pressure is to apply pressure greater than the vapor pressure of the volatile components in resin, preventing them from evolving from the resin during the curing cycle. This approach yields a denser structure free of porosity and with better mechanical properties. (Thomas et al., 2008, Potter, 2012). Gündüz et al. (1981), Gündüz and Yalçin (1987), Chen et al. (2002) have all reported the higher tensile and flexural strength samples in comparison with the samples of the vacuum process implying higher stability of the samples during the preparation phases, e.g., cutting, grinding and polishing.

Viscosity modifiers. Some authors suggested that adding solvents such as toluene or acetone lowers the viscosity of epoxy mixture and results in better impregnation. We added toluene to the epoxy mixture at three different weight ratios $5 \%, 10 \%$ and $30 \%$. We used the viscosity behavior of the different epoxy and toluene compositions, depicted in Fig. A2, to provide an estimation for the resin capillary penetration length according to Eq. (1). Fig. 8 shows no substantial change in the resin impregnation rate or volume unless toluene weight ratio is more than $30 \%$. However, the mechanical properties of the cured epoxy mixed with such $30 \%$ of toluene deteriorate severely, i.e. the lower hardness and toughness have been observed.

In addition to mechanical property deterioration, for the higher toluene ratio we observed more aggressive shrinkage type $2 \mathrm{~A}$ and $2 \mathrm{~B}$ defects seen in Fig. 9. Moreover, Fig. 9a shows a clear phase boundary between toluene and resin mixture. Therefore, we conclude that adding solvents such as toluene is not acceptable for high resolution pore casts. It does not cause a significant increase in the impregnation volume, but it causes severe defects that may lead to false interpretations. 


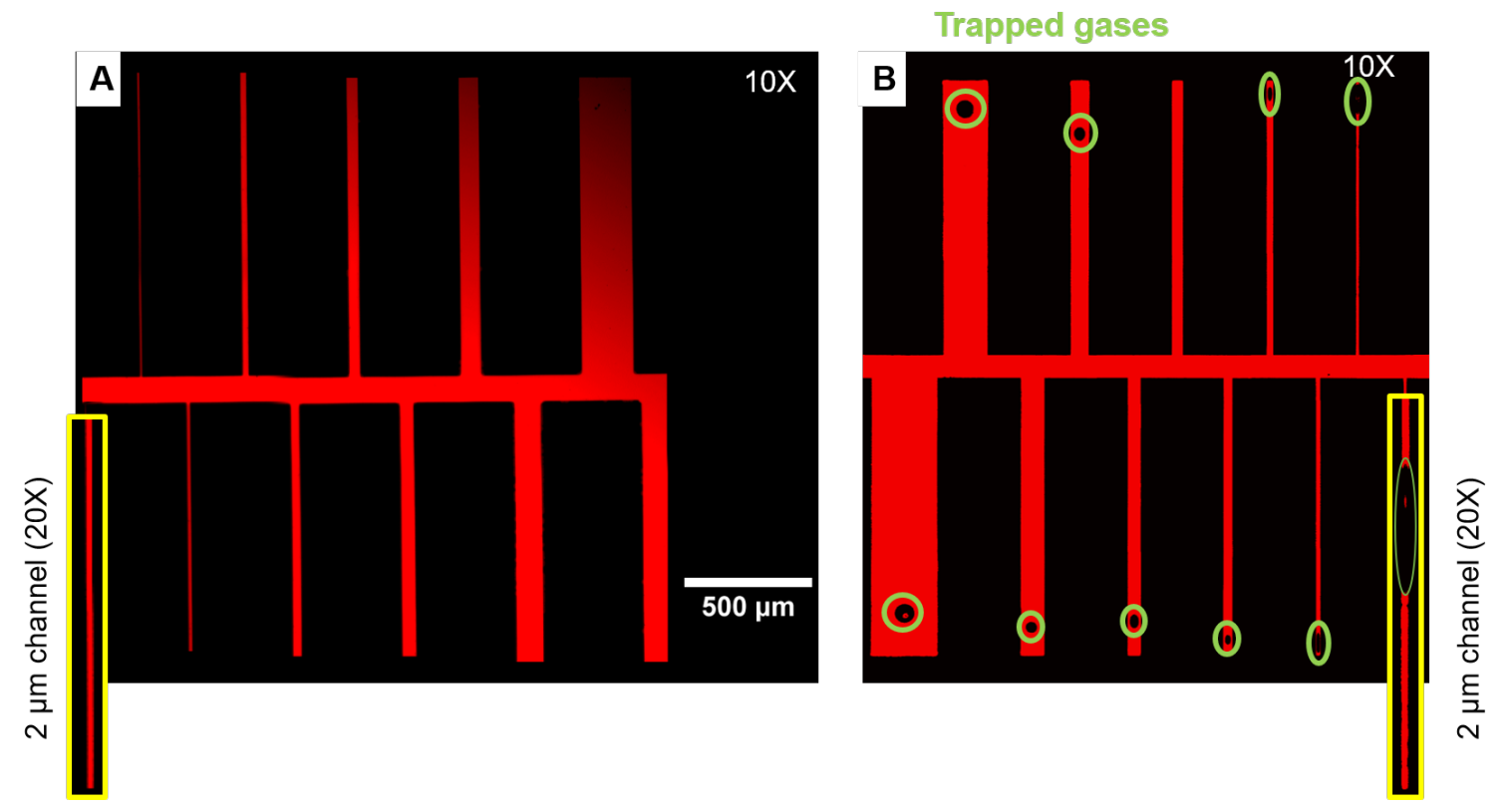

Figure 5: Confocal images of silicon micromodels impregnated by resin mixed with fluorescent dye. Impregnation is carried out by: (A) High pressure, 65 bar, preceded by a vacuum step to degas the sample. (B) Vacuum impregnation of a micromodel. Gas bubbles trapped during the inefficient impregnation are highlighted inside the channels of the vacuum.
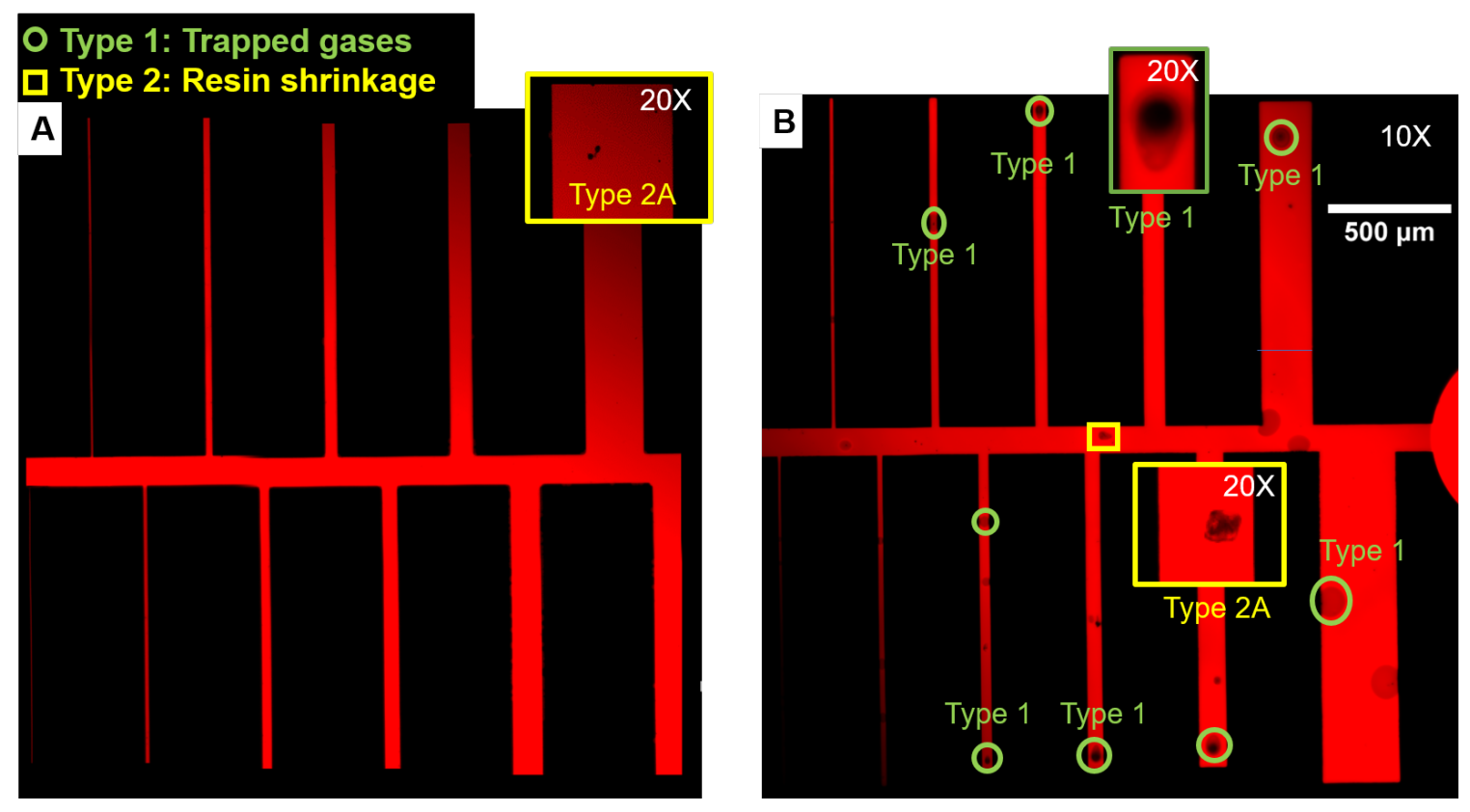

Figure 6: Confocal images of the silicon micromodels impregnated by resin mixed with fluorescent dye under: (a) High pressure preceded by degassing step, and (b) High pressure only with no prior degassing. Two types of defects were observed: type 1, gas bubbles trapped inside the model due to inefficient degassing is seen only in (b), and type 2 attributed to resin shrinkage is observed in both (a) and (b). Type $2 A$ refers to void shrinkage defects while type $2 \mathrm{~B}$ to cracks. 

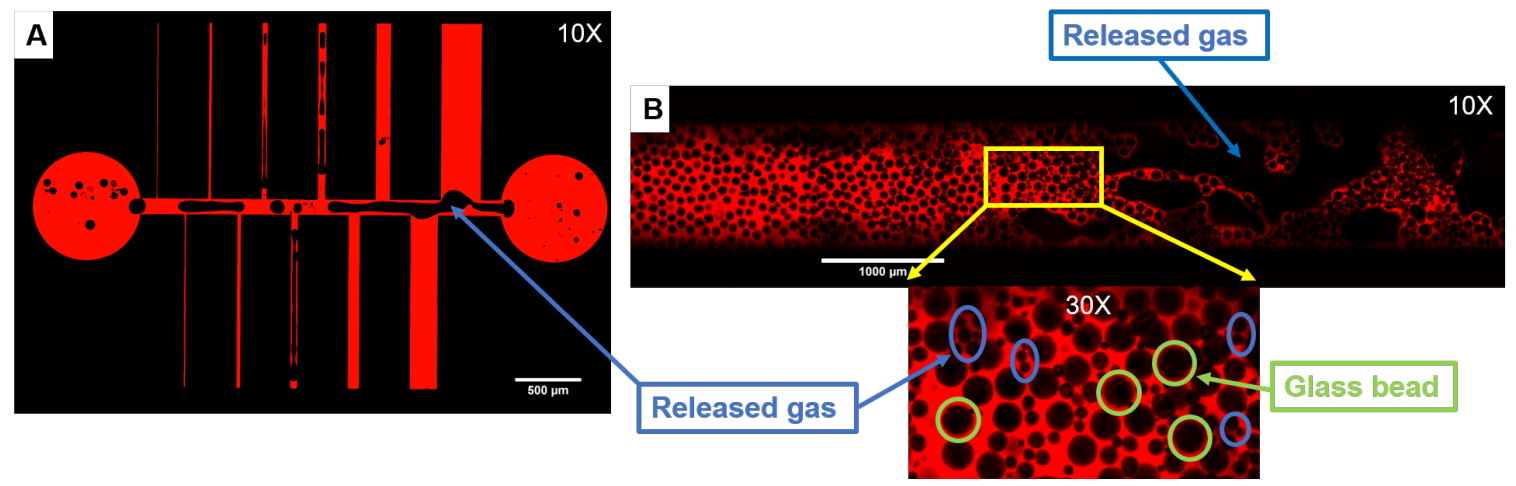

Figure 7: Confocal images of two types of the micromodels impregnated with resin under high pressure, 65 bar, released to atmospheric before complete curing took place. A) Silicon micromodel, and B) Sintered glass beads. Defects in both models occurred when nitrogen gas dissolved at 65 bar was abruptly released when the pressure was dropped to atmospheric and the resin was incompletely cured. The gas release in model $B$ was severe enough to debond some of the sintered glass beads.

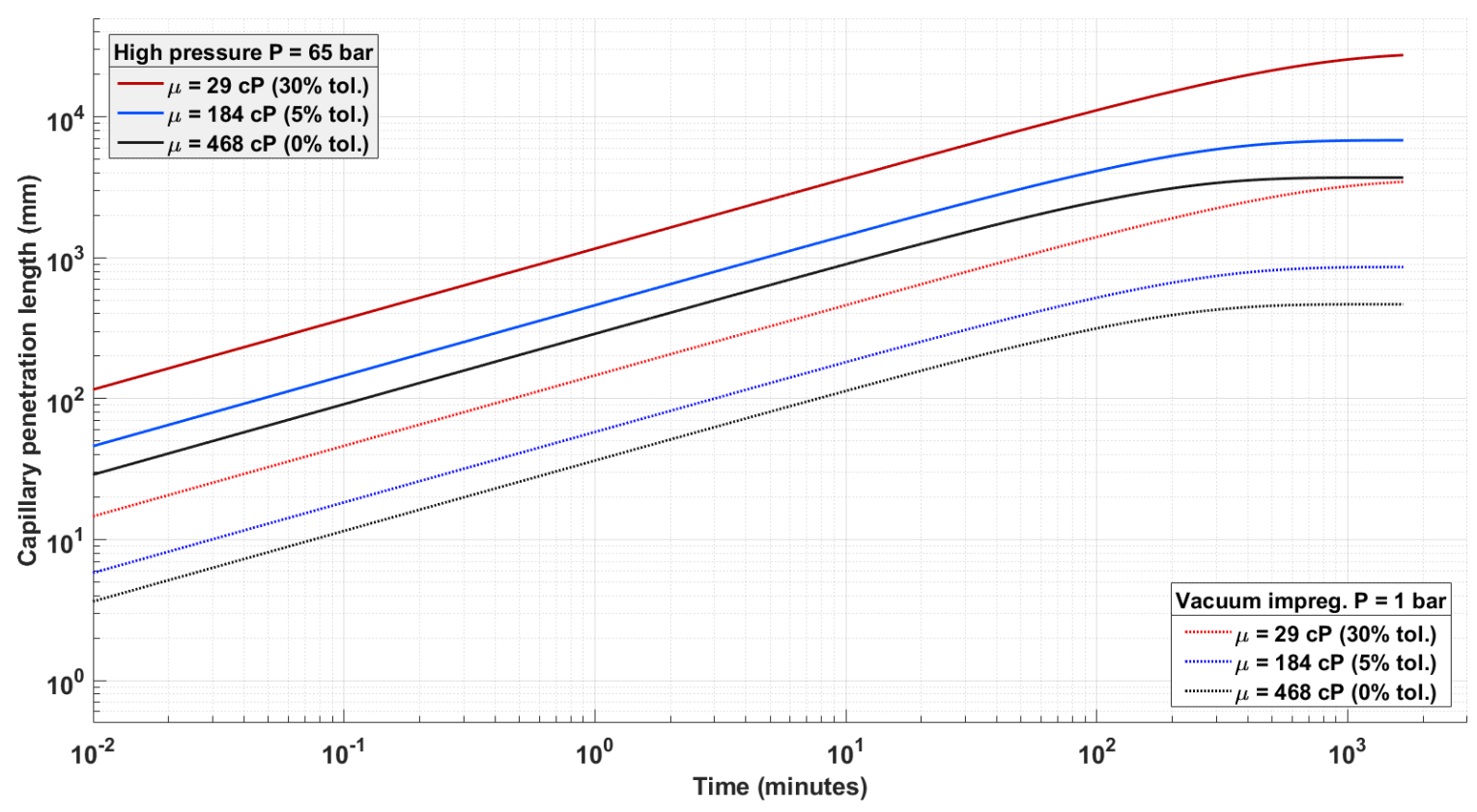

Figure 8: Plot of the estimated capillary penetration length vs. time in a capillary with the $\mathbf{4 0} \mu \mathrm{m}$ diameter. Three resin and toluene compositions were tested: (a) pure resin, (b) resin $+5 \%$ tol., (c) resin $+30 \%$ tol. Both vacuum and high pressure impregnation were considered for all compositions. The penetration length does not change significantly unless the toluene content is more than $30 \%$; however, the mechanical properties for this composition greatly deteriorate. 


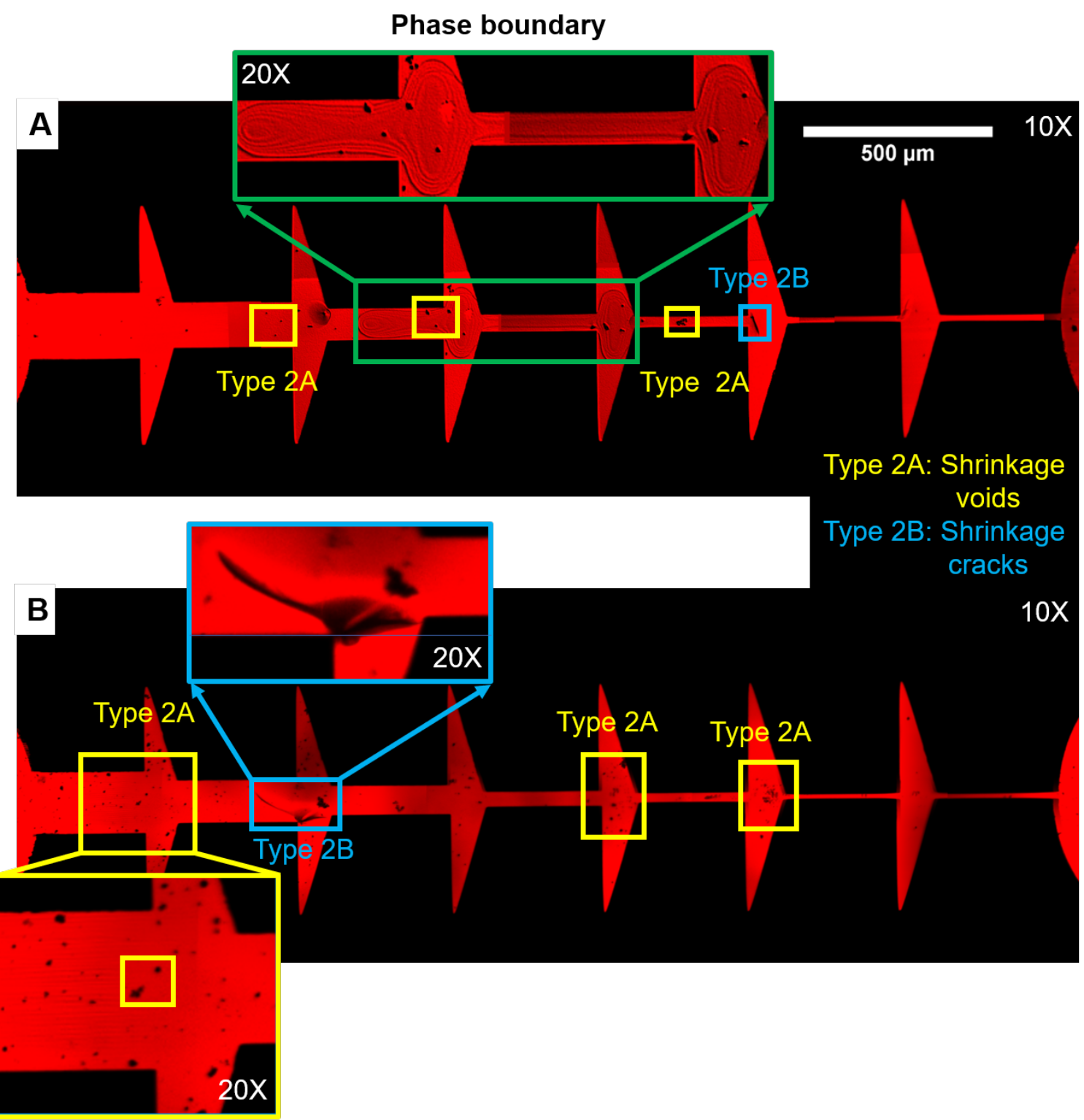

Figure 9: Confocal images of two micromodels impregnated under vacuum. Two compositions of resin and toluene were used: (A) epoxy mixed with $5 \%$ toluene, and $(B)$ epoxy mixed with $30 \%$ toluene. In model $(A)$ a phase boundary between toluene and epoxy was detected. Shrinkage defects, especially type $2 \mathrm{~A}$, is visibly more abundant in model $(B)$ where the toluene fraction was higher. 


\section{Imaging carbonate pore casts}

The various defects in pore casting, such as resin shrinkage and gas bubbles, can lead to erroneous imaging results. Quality of the high resolution confocal images greatly deteriorates where these defects are observed.

MicroCT. To evaluate quality of the cured epoxy pore casts of carbonate rock samples we have used micro-CT imaging. The micro-CT images of the samples were screened for the resin penetration depth and the aforementioned defects. We verified the homogeneity of resin impregnation throughout the entire sample thickness of $5.1 \mathrm{~mm}$, and we did not find any changes in the CT intensity values across all 943 slices, one of which is shown in Fig. 10. We are still able to identify some defects highlighted in Fig. 10; however, these defects represent only $0.21 \%$ of the total pore volume throughout the entire sample thickness. Since the voxel size is $5.41 \mu \mathrm{m}$, the microporosity is characterized only by the difference in grayscale values; however, no further information regarding its geometry and distribution inside the grain can be obtained at this resolution.

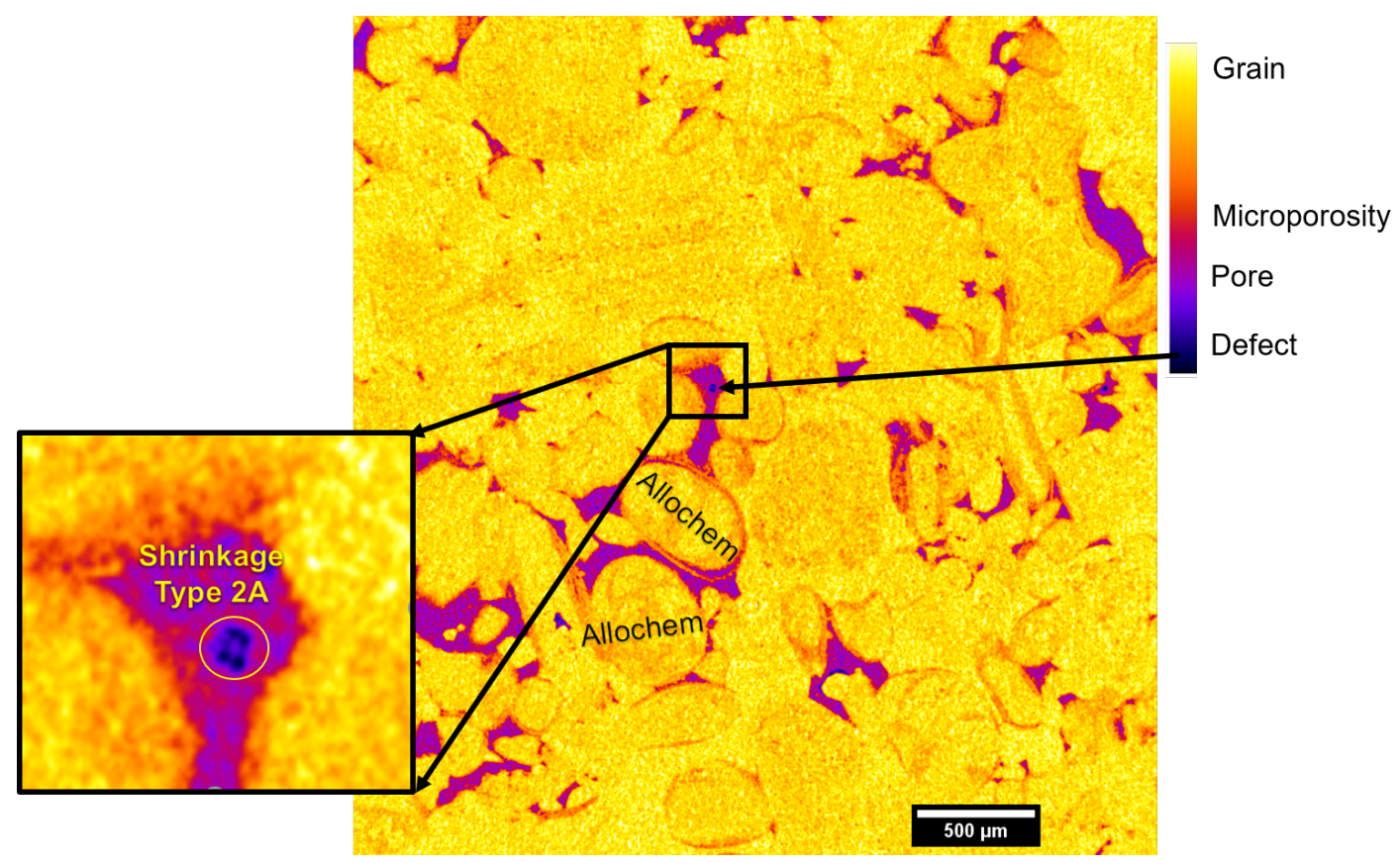

Figure 10: The micro-CT image of the Indiana limestone sample impregnated with epoxy. This grayscale image is but one slice of the 943 slices representing the $5.1 \mathrm{~mm}$ sample thickness. The sample is composed mainly of allochems as indicated; however, the microporosity geometry can hardly be identified at the resolution of $5.41 \mu \mathrm{m}$ per voxel. Defects, such as the highlighted shrinkage defect of type $2 \mathrm{~A}$, could still be identified in the impregnated sample. They represent only $0.21 \%$ of the pore volume. Colors are assigned to different grayscale values to aid visualization. The color code indicates the different elements of the image.

Confocal microscopy. Fig. 11 illustrates the confocal images of the epoxy pore casts of Indiana limestone obtained from the high pressure resin impregnation. Prior to impregnation, the rock sample was heated in the oven at $60^{\circ} \mathrm{C}$ for 12 hours. Then, the sample was degassed under vacuum of 120 mbar for about 10 minutes, followed by pressurized resin impregnation at 65 bar for 36 hours. We followed the procedure described in (Hassan et al., 2017) to image the etched epoxy pore casts of Indiana limestone with confocal microscopy. The samples were etched with dilute acid to dissolve the opaque rock matrix. This etching process rendered the residual pore cast more "transparent" to CLSM. We have achieved the depths of investigation of up to a few hundreds of micrometers. The high resolution 3D CLSM images of the pore cast were helpful in identifying the allochems and the interparticle porosity (IP), which is made up of the relatively large pores shown in Fig. 11a. The micrite cement (MC) coating the allochem rims along with the intergranular microporosity (IGM) could be identified using the 20X-water immersion objective, see Fig. 11b. These details could not observed in the microCT images. 

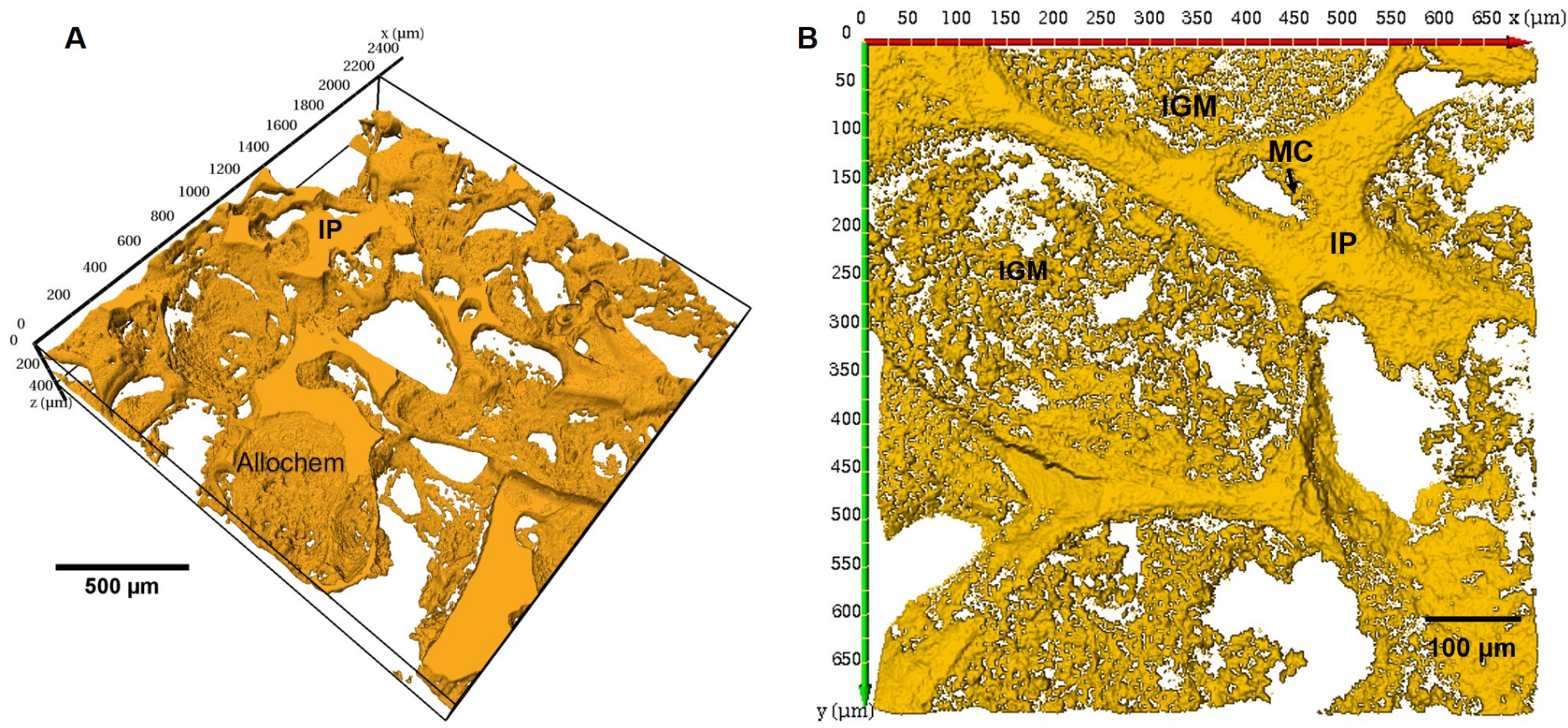

Figure 11: Confocal images of the etched epoxy pore cast of Indiana limestone, where the solid represents the pore space and the etched away grains are white. Sample (A) was imaged with the $10 \mathrm{X}$ air objective and the resolution of $1.6 \mu \mathrm{m}$ per pixel for the total size of $2.3 \mathrm{~mm} \times 2.3 \mathrm{~mm} \times 0.33 \mathrm{~mm}$. Sample (B) was imaged with the $20 \mathrm{X}$ water immersion objective and the resolution of $0.34 \mu \mathrm{m}$ per pixel for the total size of $0.7 \mathrm{~mm} \times 0.7 \mathrm{~mm} \times 0.9 \mathrm{~mm}$. The higher resolution CLSM images allowed detecting types of microporosity: the micritic intragranular microporosity (IGM) can be seen within the grains. The micrite cement (MC) can be seen along the rims of allochems. The inter-particle (IP) pores are the relatively large voids at the junctions of the allochems.

\section{Conclusions}

In this study we have developed and optimized a high pressure resin impregnation protocol that produces the high-quality pore casts and captures the complex pore space of micritic carbonates. For the first time, we have used silicon micromodels as a reference to validate the parameters of the high-pressure resin impregnation for the subsequent high-resolution 3D confocal imaging of micritic pore space.

From experiment and by using a simple theoretical model we showed that applying a high pressure of 65 bars results in a more efficient resin impregnation. We also observed the impact of high pressure on the rate of epoxy curing reactions and on the mechanical properties of the pore casts.

We have demonstrated that the low linear shrinkage of the resin, usually $1-2 \%$, might be misleading because it does not prevent the defects that may occur during resin curing. Two types of shrinkage defects were identified; voids formed within the resin, and cracks developed across the resin structure. The shrinkage defects were found to be more aggressive when degassing was performed under higher vacuum levels or for a longer time. Diluting resin with solvents such as toluene to reduce the resin viscosity caused aggressive defects and deteriorated the optical and mechanical properties of the cured resin.

Based on the experiments with micromodels, we have optimized the procedure for producing the high quality epoxy pore casts suitable for high resolution imaging. We have used this procedure to characterize the pore space of Indiana limestone. Micro-CT images of the resin-impregnated Indiana limestone samples have shown that the resin impregnates the samples uniformly. The micro-CT images have also shown that some minor defects persist, but these defects represent only $0.21 \%$ of the sample's pore volume. The CLSM images of the impregnated samples highlighted different types of the microporosity, such as the intragranular microporosity and the micritic cement. 


\section{Appendix A. Methods Silicon micromodels}

Lithography. Single-side polished, P-type, $<1,0,0\rangle$, four-inch silicon wafers with a thickness of $525 \mu \mathrm{m}$, procured from LUVA System Inc., were used in this study. First, the silicon wafers were spin-coated with a positive photoresist, AZ 5214, procured from Merck Performance Materials $\mathrm{GmbH}$, at the speed of $2800 \mathrm{r} / \mathrm{min}$ for $40 \mathrm{~s}$, to make the photoresist reach a thickness of around $1.6 \mu \mathrm{m}$. Second, the coated wafers were prebaked on hot plates at the temperature of $110^{\circ} \mathrm{C}$ for 2 minutes. Third, the Heidelberg $\mu \mathrm{PG} 501$ was used for direct-writing of custom patterns, shown in Fig. A1a, on the silicon wafers using a laser with 390 nm wave-length and exposure time of $55 \mathrm{~ms}$. Finally, the wafers were developed in AZ $726 \mathrm{MIF}$, procured from Microchemicals GmbH, for 1 minute.

Deep Reactive Ion Etching (DRIE). Etching the patterns into the silicon models was carried out using DRIE following Bosch procedure (Lärmer and Schilp, 1994), in which the etching and passivation steps are used alternatively to vertically etch the silicon wafers. We used the Plasmalab System 100 provided by Oxford Instruments. The passivation step parameters are

1. ICP Power: 1300 Watts,

2. Pressure: 30 mTorr,

3. Temperature: $-20^{\circ} \mathrm{C}$,

4. $\mathrm{C}_{4} \mathrm{~F}_{8}$ flow rate: 100 standard cubic $\mathrm{cm}$ per minute,

5. $\mathrm{SF}_{6}$ flow rate: 5 standard cubic $\mathrm{cm}$ per minute,

while the parameters for etching are, ICP Power: 1300 Watts, Pressure: 30 mTorr, Temperature: $-20{ }^{\circ} \mathrm{C}, \mathrm{C}_{4} \mathrm{~F}_{8}$ flow rate: 5 standard cubic $\mathrm{cm}$ per minute, $\mathrm{SF}_{6}$ flow rate: 100 standard cubic $\mathrm{cm}$ per minute.

After running the etching and passivation steps alternatively for 180 times for about 45 minutes, approximately $100 \mu$ m of the engraved pattern was etched in the silicon wafer. Afterwards, another run is required to etch the inlets through the entire wafer thickness. Finally the silicon micromodel was sealed from one end with a glass wafer using anodic bonding.

\section{Sintered glass beads}

Class V soda lime glass spheres of 75-90 $\mu \mathrm{m}$ size were procured from Mo-Sci specialty products, L.L.C. The beads were packed inside a $1 \mathrm{~mm}$ square quartz capillary sealed from one end. The capillary was then loaded to an oven preheated to $870{ }^{\circ} \mathrm{C}$. The capillary was kept inside the oven for only 3 minutes, which gave enough time for the beads to be sintered. Longer heating periods caused melting. Fig. A1b shows the final sintered glass beads model.
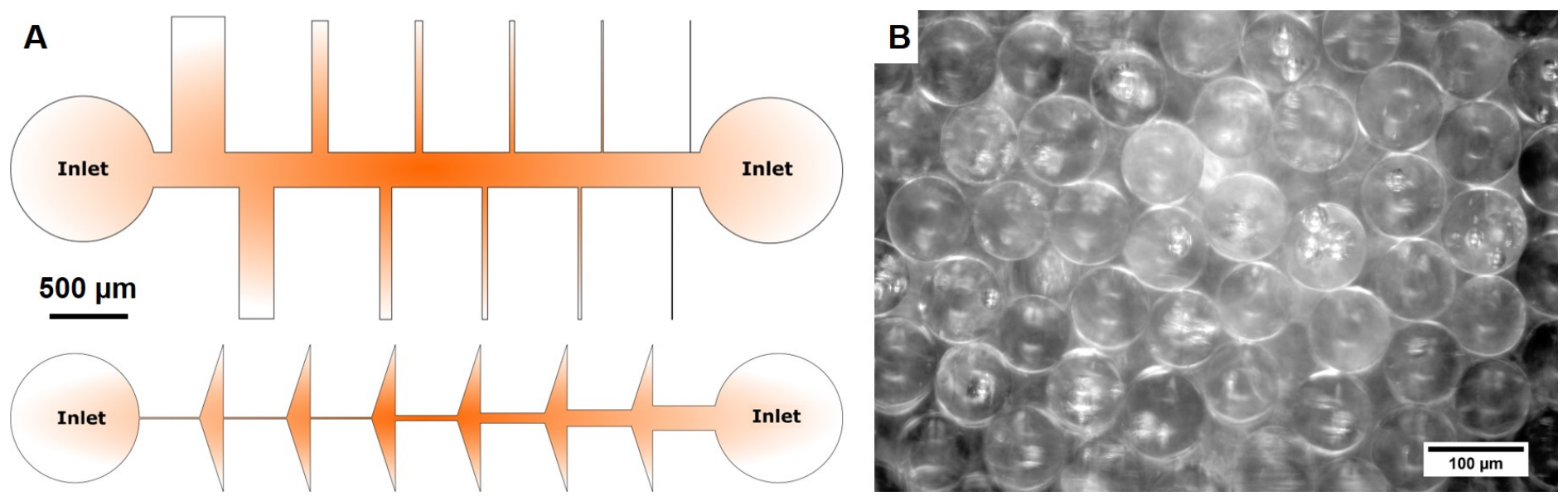

Figure A1: The fabricated micromodels used to study the epoxy impregnation parameters. (A) Two patterns engraved in silicon micromodels. (B) The sintered glass beads inside a $1 \mathrm{~mm}$ square glass capillary imaged with optical microscope. 


\section{Materials}

Resin and chemicals. For impregnation, we used a two-component amine-cured epoxy resin R3501/H5064 (part A polymer and part $\mathrm{B}$ hardener) with the mixture viscosity of $468 \mathrm{cP}$, and glass transition temperature of $54-57^{\circ} \mathrm{C}$ procured from EpicResins Inc. The fluorochrome used to dye the epoxy was Rhodamine-B, procured from Alfa Aesar. This particular epoxy resin and dye were selected after testing a number of combinations of commercially available products. The fluorescence excitation and emission wavelengths of Rhodamine-B are in the range of 514-568 nm and 570-600 nm, respectively. Rhodamine-B was dissolved in part A of the epoxy with a weight ratio 1:200 by stirring with magnetic stirrer for an hour until complete dissolution. Then part B was added to the solution usually with $25 \%$ excess to what was recommended by the supplier, to avoid developing shrinkage defects due to the low conversion ratio, caused by the evaporation during application of the vacuum, when the resin vitrifies as discussed earlier.

Rock samples. We used the well-known Indiana limestone for rock impregnation. Indiana limestone is of the Mississippian age and is quarried from the Salem Formation, Indiana (Patton and Carr, 1982). Indiana limestone can be classified as a fossiliferous, calcitecemented grainstone predominantly composed of pellets, moderately rounded skeletal grains, ooids, and shell fragments. The allochems are coated with micritic cement, which comprises equant sparry calcite crystals. The pore system in the Indiana limestone rock type used in this study is commonly dominated by the well-connected intergranular pores (Churcher et al., 1991, Vajdova et al., 2012), which constitute the pore space between the allochems. The micritic material of the pore-lining micritic cement is highly microporous (MC). Micropores are also observed within the microcrystalline material of the allochems resulting in intragranular microporosity (IGM).

\section{Viscosity measurement}

We measured the viscosity of the pure resin. Also, we tested the effect of diluting the resin with toluene at three different weight ratios, $5 \%, 10 \%$ and $30 \%$. The viscosity was measured using an electromagnetic viscometer, EMS 1000, provided by KEM kyoto Co. Ltd., for 3 hours. Then the constant a and b in Eq. (2) were determined for every mixture so that the viscosity behavior could be extrapolated, as shown in Fig. A2.

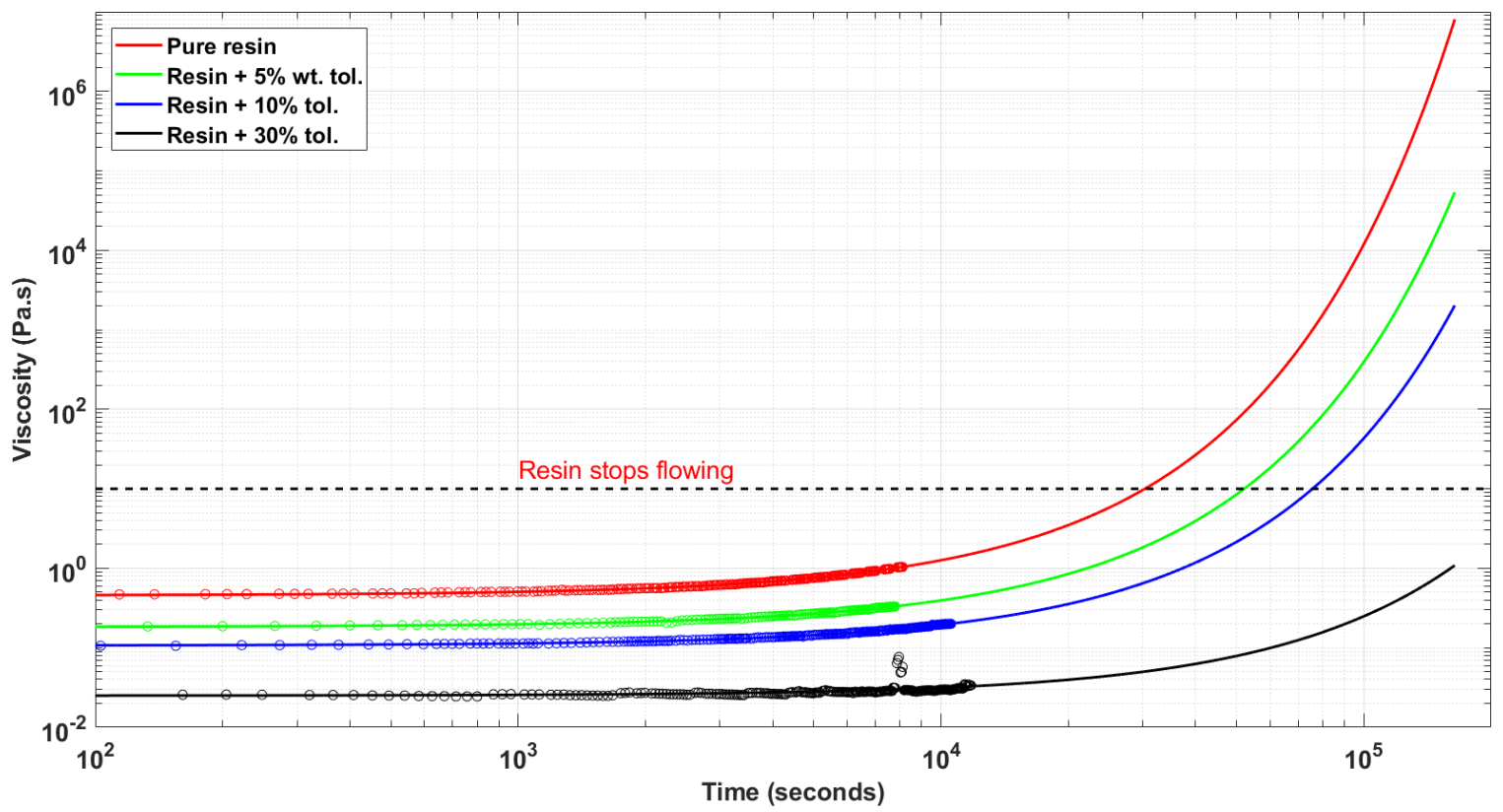

Figure A2: Plot of the resin viscosity vs. time. The gelation behavior is measured with a viscometer, under an average shear rate of $4 s^{-1}$, for 3-4 hours, and to extrapolate the behavior Eq. (2) was fitted. The viscosity is measured for 4 resin and toluene compositions: (a) pure resin, (b) resin $+5 \%$ wt. tol., (c) resin $+10 \%$ wt. tol., and (d) resin $+30 \%$ wt. tol. Composition (d) experienced viscosity reduction of almost 15 times; however, the curing time nearly doubled and the mechanical properties greatly suffered.

\section{Pore throat size measurement}

The MIP results, plotted in Fig. A3, indicate the bi-modal pore types with the median and minimum pore throat diameter of $39.1 \mu \mathrm{m}$ and $0.04 \mu \mathrm{m}$, respectively. This bimodality is due to the contribution of MC created by fine calcite crystals lining the pores and the IGM present in the allochems, mainly oolites.

\section{Vacuum impregnation and degassing}

A desiccator with a tilting handle, shown in Fig. A4a, is connected to a pump for pulling vacuum and degassing the epoxy and the 


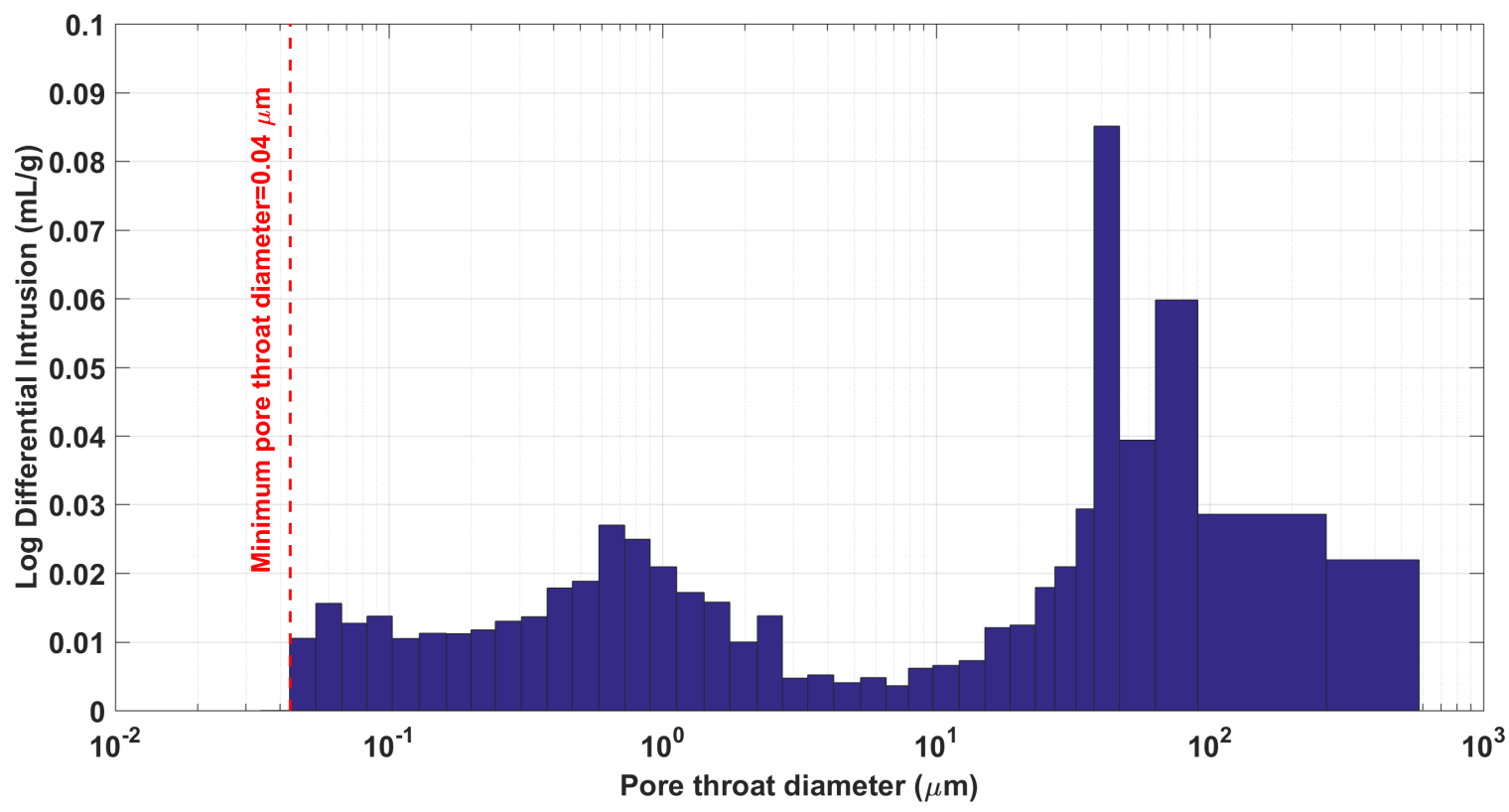

Figure A3: The pore throat size distribution based on the MIP data represented by a plot of the log-differential of mercury volume intrusion $(\mathrm{mg} / \mathrm{L})$ vs. the pore throat diameter $(\mu \mathrm{m})$.

impregnated material. The vacuum impregnation unit model is LSSA-011, procured from Pace Technologies. For the vacuum level of 10 mbar and higher we used a vacuum oil pump model 15501 procured from Robinair, and for the vacuum level of 120 mbar and higher we used in house vacuum. The sample and the epoxy mixture were vacuumed together in one chamber without being in direct contact. For the vacuum level of 120 mbar and higher the degassing procedure typically lasted for 15 minutes. For the vacuum level below 10 mbar, the duration was approximately 5 minutes. Otherwise the epoxy would boil quite aggressively. Then the epoxy was poured over the sample contained inside a disposable mold.

\section{High pressure impregnation}

Once the degassing procedure was completed, samples were immediately transferred into a high pressure vessel, shown in Fig. A4b, and slowly pressurized at a rate of $50 \mathrm{psi} / \mathrm{min}$ to $1000 \mathrm{psi}(6.5 \mathrm{MPa})$ by G6 nitrogen. The desired pressure was reached via a high delivery pressure regulator (Air liquide, model Q1-208CS-CGA580). The high pressure vessel is a pipe cell procured from MetaRock Laboratories that has $60 \mathrm{~mm}$ in diameter and $190 \mathrm{~mm}$ in height, made of 17-4 PH annealed stainless steel, and tested up to $13000 \mathrm{psi}$ (89.7 MPa). The sample was kept under high pressure until the resin was completely cured; otherwise, excessive bubbles would emerge, as discussed earlier in Fig. 7.

\section{Sample preparation}

After the resin had cured, the vacuum- or high pressure-impregnated samples were released from the disposable molds. The excess resin surrounding the sample was removed using M-Prep $5^{\mathrm{TM}}$ Grinder/Polisher from Allied High Tech. First, the sample was ground by silicon carbide abrasive paper (320 grit size) for 1 minute, followed by 3 minutes and 5 minutes on grit size 600 and 1200 , respectively. The final polishing was done with a polishing cloth (Red final C) and a $0.04 \mu \mathrm{m}$ colloidal silica suspension. Once polished, the samples were visually inspected using the Olympus BX61 optical materials microscope for defects, like bubbles, micro-fractures, as well as for polishing quality.

\section{Imaging}

CLSM imaging. The impregnated samples were then imaged using the Zeiss LSM 880 confocal microscope. An argon 514 nm laser was used to excite Rhodamine-B. All impregnated micromodels were imaged by the 10X plan apochromat objective lens with numerical aperture (NA) of 0.45 . The epoxy pore casts of Indiana limestone were imaged using the aforementioned lens and for higher magnification the $20 \mathrm{X}$ plan apochromat water-immersion objective lens with NA of 1 . The photomultiplier gain was set between 450-580, while the laser intensity used was kept between 2.5-3.5 for all the samples. The tile scanning module in Zen software enabled imaging of samples across the entire investigation area. Stitching was implemented on the tiles with a 12-15\% overlap.

Micro-Computed Tomography (Micro-CT). The impregnated epoxy pore cast of Indiana limestone was imaged using uniTOM system provided by XRE. The system is a closed type X-ray tube with energy level 40-130 kV/39 W, and the acquired voxel size was $5.41 \mu \mathrm{m}$. 

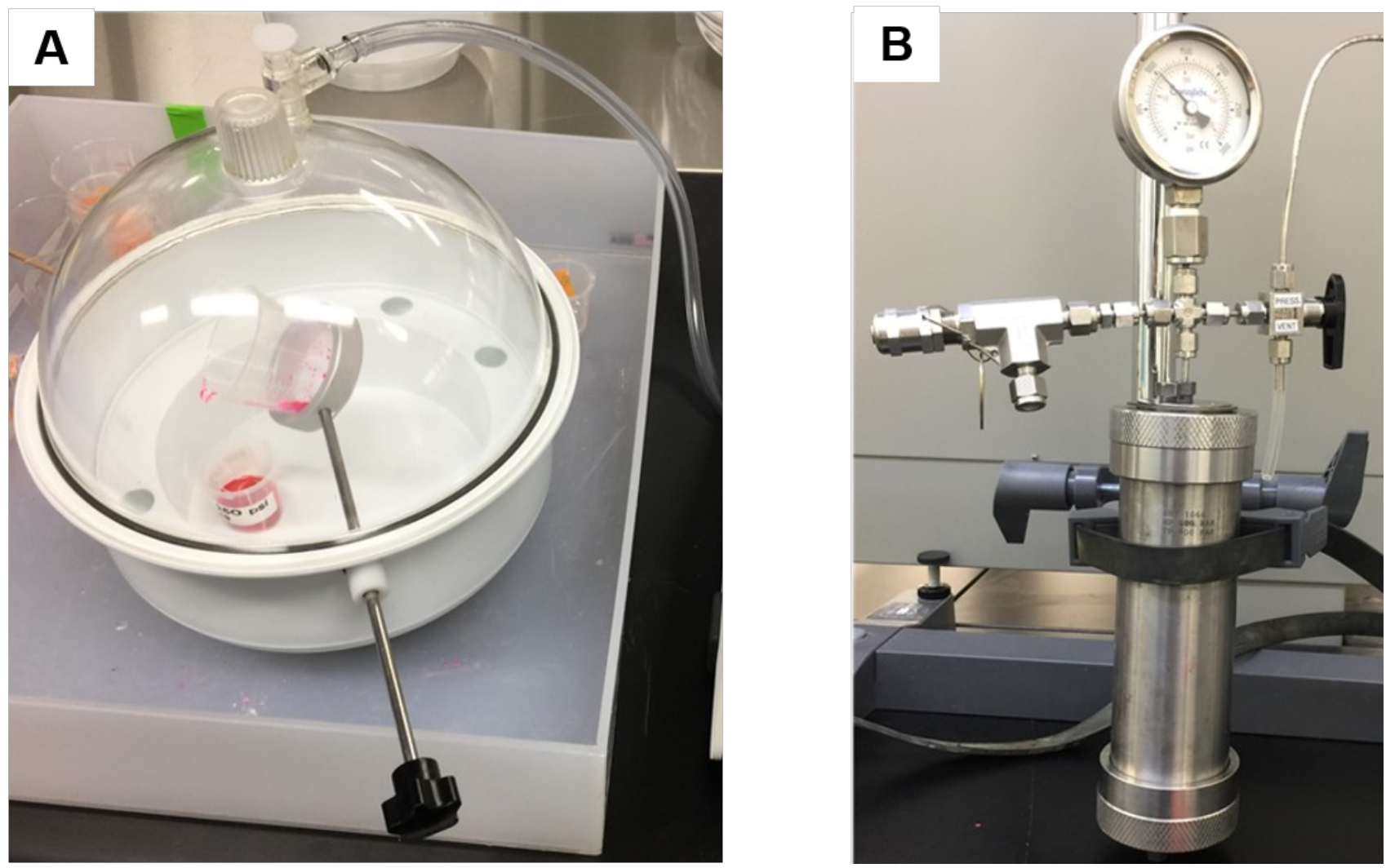

Figure A4: The equipment used for resin impregnation. (A) The desiccator with a tilting handle used for degassing the sample and epoxy separately when connected to a vacuum source. (B) The high pressure vessel connected to high pressure gas line.

\section{Acknowledgments}

We thank Dr. Marijn Boone from Tescan-XRE for acquiring the micro-CT images. We thank Mr. Darweesh for the fruitful discussions on the epoxy curing mechanism. We are grateful to the Nanofabrication Core Lab team at KAUST, especially to Dr. Ulrich Buttner and Mr. Ahad Syed, for their support in fabricating the silicon micromodels. This project has been funded by KAUST through the Ali I. Al-Naimi Petroleum Engineering Research Center (ANPERC).

\section{References}

Beckett, D. and Sellwood, B. (1991). A simple method for producing high-quality porecasts of carbonate rocks. Sedimentary Geology, 71(1-2):1-4.

Boës, X. and Fagel, N. (2005). Impregnation method for detecting annual laminations in sediment cores: an overview. Sedimentary geology, 179(3-4):185-194.

Camuti, K. S. and McGuire, P. T. (1999). Preparation of polished thin sections from poorly consolidated regolith and sediment materials. Sedimentary Geology, 128(1-2):171-178.

Cantrell, D. L., Hagerty, R. M., et al. (1999). Microporosity in arab formation carbonates, saudi arabia. GeoArabia, 4(2):129-154.

Chen, J., Zampini, D., and Walliser, A. (2002). High-pressure epoxy-impregnated cementitious materials for microstructure characterization. Cement and concrete research, 32(1):1-7.

Churcher, P., French, P., Shaw, J., Schramm, L., et al. (1991). Rock properties of berea sandstone, baker dolomite, and indiana limestone. In SPE International Symposium on Oilfield Chemistry. Society of Petroleum Engineers.

Eom, Y., Boogh, L., Michaud, V., Sunderland, P., and Månson, J.-A. (2001). Stress-initiated void formation during cure of a threedimensionally constrained thermoset resin. Polymer Engineering \& Science, 41(3):492-503.

Fredrich, J. (1999). 3d imaging of porous media using laser scanning confocal microscopy with application to microscale transport processes. Physics and Chemistry of the Earth, Part A: Solid Earth and Geodesy, 24(7):551-561. 
Fredrich, J., Menendez, B., and Wong, T.-F. (1995). Imaging the pore structure of geomaterials. Science, 268(5208):276-279.

Fullmer, S. M., Guidry, S. A., Gournay, J., Bowlin, E., Ottinger, G., Al Neyadi, A., Gupta, G., Gao, B., and Edwards, E. (2014). Microporosity: Characterization, distribution, and influence on oil recovery. In IPTC 2014: International Petroleum Technology Conference.

Gies, R. et al. (1987). An improved method for viewing micropore systems in rocks with the polarizing microscope. SPE Formation Evaluation, 2(02):209-214.

Gündüz, G. and Yalçin, N. (1987). Strength of steel-fibre-reinforced polymer concrete: Effect of impregnation technique. Composites science and technology, 30(2):127-135.

Gündüz, G., Yeter, B., Tuğlu, P., and Ahmed, I. (1981). Styrene-acrylonitrile co-polymer impregnated mortar. Journal of Materials Science, 16(1):221-225.

Hamraoui, A. and Nylander, T. (2002). Analytical approach for the lucas-washburn equation. Journal of colloid and interface science, 250(2):415-421.

Hassan, A., Chandra, V., Yutkin, M., Patzek, T., Espinoza, D., et al. (2017). Improving pore network imaging \& characterization of microporous carbonate rocks using multi-scale imaging techniques. In SPE Abu Dhabi International Petroleum Exhibition \& Conference. Society of Petroleum Engineers.

Head, M. and Buenfeld, N. (2006). Confocal imaging of porosity in hardened concrete. Cement and concrete research, 36(5):896-911.

Hurst, A. and Nadeau, P. H. (1995). Clay microporosity in reservoir sandstones: an application of quantitative electron microscopy in petrophysical evaluation. AAPG bulletin, 79(4):563-573.

Jim, C. (1985). Impregnation of moist and dry unconsolidated clay samples using spurr resin for microstructural studies. Journal of Sedimentary Research, 55(4).

Jobe, T., Geiger, S., Jiang, Z., and Agar, S. (2018). Micropore network modelling from 2d confocal imagery: impact on reservoir quality and hydrocarbon recovery. Petroleum Geoscience, 24(3):323-334.

John, D. S. (1990). The use of large-area thin sectioning in the petrographic examination of concrete. In Petrography applied to concrete and concrete aggregates. ASTM International.

Kemp, A. (1990). Sedimentary fabrics and variation in lamination style in peru continental margin upwelling sediments. In Proc. ODP, Sci. Results, volume 112, pages 43-58.

Kjellsen, K., Monsøy, A., Isachsen, K., and Detwiler, R. (2003). Preparation of flat-polished specimens for sem-backscattered electron imaging and x-ray microanalysisŮimportance of epoxy impregnation. Cement and concrete research, 33(4):611-616.

Klaver, J., Hemes, S., Houben, M., Desbois, G., Radi, Z., and Urai, J. (2015). The connectivity of pore space in mudstones: insights from high-pressure wood's metal injection, bib-sem imaging, and mercury intrusion porosimetry. Geofluids, 15(4):577-591.

Lärmer, F. and Schilp, A. (1994). Verfahren zum anisotropen ätzen von silizium. Deutsches Patent DE, $42(41): 045$.

Levine, S., Reed, P., Watson, E., and Neale, G. (1976). A theory of the rate of rise of a liquid in a capillary. In Colloid and Interface Science, Volume III, pages 403-419. Elsevier.

Li, M., Zhang, Z. G., and Sun, Z. J. (2006). Contact angle of epoxy resin measured by capillary impregnation and the wilhelmy technique. Polymers and polymer composites, 14(3):251.

Maija Leskinen, A. (1987). Layer structure in model coatings. Tappi journal, 70(12):101-106.

Marmur, A. (1992). Penetration and displacement in capillary systems of limited size. Advances in Colloid and interface Science, 39:13-33.

Minoura, N. and Conley, C. (1971). Technique for impregnating porous rock samples with low-viscosity epoxy resin. Journal of Sedimentary Research, 41(3):858-861.

Morrow, N. R. and Buckley, J. (2006). Wettability and oil recovery by imbibition and viscous displacement from fractured and heterogeneous carbonates. Chemical \& Petroleum Engineering. 
Muller, M. (2006). Introduction to confocal fluorescence microscopy, volume 69. SPIE press.

Patton, J. B. and Carr, D. D. (1982). The salem limestone in the indiana building-stone district. Technical report, Indiana Geological Survey.

Pawley, J. (2010). Handbook of biological confocal microscopy. Springer Science \& Business Media.

Petford, N., Davidson, G., and Miller, J. (2001). Investigation of the petrophysical properties of a porous sandstone sample using confocal scanning laser microscopy. Petroleum Geoscience, 7(2):99-105.

Pittman, E. D. and Duschatko, R. W. (1970). Use of pore casts and scanning electron microscope to study pore geometry. Journal of Sedimentary Research, 40(4):1153-1157.

Plepys, A. and Farris, R. (1990). Evolution of residual stresses in three-dimensionally constrained epoxy resins. Polymer, 31(10):19321936.

Potter, K. (2012). Resin transfer moulding. Springer Science \& Business Media.

Poulin, N., Tanguy, P., Aspler, J., and Larrondo, L. (1997). Numerical and physical modeling of the permeability of paper to cmc and coating liquids. The Canadian Journal of Chemical Engineering, 75(5):949-955.

Ramos, J., Pagani, N., Riccardi, C., Borrajo, J., Goyanes, S., and Mondragon, I. (2005). Cure kinetics and shrinkage model for epoxy-amine systems. Polymer, 46(10):3323-3328.

Rothwell, R. G. and Rack, F. R. (2006). New techniques in sediment core analysis: an introduction. Geological Society, London, Special Publications, 267(1):1-29.

Shah, S., Crawshaw, J., and Boek, E. (2014). Preparation of microporous rock samples for confocal laser scanning microscopy. Petroleum Geoscience, 20(4):369-374.

Shah, S. M. K. (2014). Multi-scale imaging of porous media and flow simulation at the pore scale. $\mathrm{PhD}$ thesis, Imperial College London.

Smith, S. J. and Anderson, R. S. (1995). A method for impregnating soft sediment cores for thin-section microscopy. Journal of Sedimentary Research, 65(3).

Soeder, D. J. (1990). Applications of fluorescence microscopy to study of pores in tight rocks (1). AAPG Bulletin, 74(1):30-40.

Spurr, A. R. (1969). A low-viscosity epoxy resin embedding medium for electron microscopy. Journal of ultrastructure research, 26(1-2):31-43.

Szekely, J., Neumann, A., and Chuang, Y. (1971). The rate of capillary penetration and the applicability of the washburn equation. Journal of Colloid and Interface Science, 35(2):273-278.

Thomas, S., Bongiovanni, C., and Nutt, S. (2008). In situ estimation of through-thickness resin flow using ultrasound. Composites Science and Technology, 68(15-16):3093-3098.

Vajdova, V., Baud, P., Wu, L., and Wong, T.-f. (2012). Micromechanics of inelastic compaction in two allochemical limestones. Journal of Structural Geology, 43:100-117.

Waldo, A. W. and Yuster, S. T. (1937). Method of impregnating porous materials to facilitate pore studies. AAPG Bulletin, 21(2):259-267.

Wardlaw, N. (1976). Pore geometry of carbonate rocks as revealed by pore casts and capillary pressure. AAPG Bulletin, 60(2):245-257.

Washburn, E. W. (1921). The dynamics of capillary flow. Physical review, 17(3):273.

Wong, H. and Buenfeld, N. (2006). Patch microstructure in cement-based materials: Fact or artefact? Cement and Concrete Research, 36(5):990-997.

Yadav, G., Dullien, F., Chatzis, I., Macdonald, I., et al. (1987). Microscopic distribution of wetting and nonwetting phases in sandstones during immiscible displacements. SPE Reservoir Engineering, 2(02):137-147.

Yamaki, J.-I. and Katayama, Y. (1975). New method of determining contact angle between monofilament and liquid. Journal of Applied Polymer Science, 19(10):2897-2909. 
Yanguas, J. and Dravis, J. J. (1985). Blue fluorescent dye technique for recognition of microporosity in sedimentary rocks. Journal of Sedimentary Research, 55(4).

Yanguas, J. and Paxton, S. T. (1986). A new technique for preparation of petrographic thin sections using ultraviolet-curing adhesive. Journal of Sedimentary Research, 56(4). 